\title{
Orbital Metastasis as Primary Presentation of Misdiagnosed Breast Cancer: A Case Report
}

\author{
Lazareva $\mathrm{E}^{1 *}$, Smichkoska $\mathrm{S}^{1}$, Atanasov Z $\mathrm{Z}^{2}$, Krstevska $\mathbf{V}^{3}$, Klisarovska $\mathbf{V}^{4}$ and Iljovska $\mathbf{M}^{1}$ \\ ${ }^{1}$ Department of Breast Cancer, University Clinic of Radiotherapy and Oncology \& Ss. Cyril and Methodius University in Skopje, Republic of North Macedonia \\ ${ }^{2}$ Department of Lung Cancer, University Clinic of Radiotherapy and Oncology \& Ss. Cyril and Methodius University in Skopje, Republic of North Macedonia \\ ${ }^{3}$ Department of Head and Neck Cancer, University Clinic of Radiotherapy and Oncology \& Ss. Cyril and Methodius University in Skopje, Republic of North
} Macedonia

${ }^{4}$ Department of Gynecologic Oncology, University Clinic of Radiotherapy and Oncology \& Ss. Cyril and Methodius University in Skopje, Republic of North Macedonia

Submission: October 28, 2019; Published: November 15, 2019

*Corresponding author: Emilija Lazareva, Department of Breast Cancer, University Clinic of Radiotherapy and Oncology, Majka Tereza 17, 1000 Skopje, Macedonia

\begin{abstract}
We report the case of orbital metastasis from a hormone-positive infiltrating lobular carcinoma in a previously misdiagnosed metastatic breast cancer in a 61-year old woman presenting with ophthalmic symptoms (diplopia, bulb proptosis). She received external beam radiotherapy, particularly 3D conformal radiotherapy, chemotherapy and hormone therapy. The metastatic involvement of the orbit in malignant tumors is a rarely diagnosed condition. Breast cancer accounts for the majority of these cases. This article highlights the importance of evaluating the one such unique metastatic site (orbital metastasis) as the first indication of advanced malignancy and reinforces the importance of an early correct diagnosis.
\end{abstract}

Keywords: Blood pressure; Baseline echocardiography; Mitral valve prolapse; Mitral regurgitation

\section{Introduction}

Breast cancer is a histologically heterogeneous disease. Infiltrating ductal carcinoma (IDC) accounts for approximately $90 \%$ of breast cancers, whereas infiltrating lobular carcinoma (ILC), the second most common breast carcinoma, comprises approximately $10 \%$ of breast cancers [1,2]. The histopathologic appearance of ILC is different from that of IDC. Whereas IDC often metastasizes to the lung, liver, bone, and brain, ILC tends to spread to the gastrointestinal tract, genitourinary tract, peritoneum, retroperitoneum, and leptomeninges [2-7]. Some investigators have suggested that, compared with IDC, ILC has a higher distant metastasis rate $[3,4,8]$, likely because of its infiltrative nature. It has been postulated that in ILC loss of E-cadherin, the cell-to-cell adhesion molecule, facilitates the metastasis process. Like ILC in the breast, metastatic ILC tends to infiltrate the affected organs in a diffuse process instead of forming a discrete tumor nodule.

The high incidence of breast cancer ocular metastasis may be related to the longer life expectancy of metastatic breast cancer patients, thus providing a longer time for intraocular metastasis to develop. It is therefore important to recall this diagnosis. The majority of symptomatic patients note a decreased visual acuity at the time of presentation. Other presenting signs or symptoms include ptosis, eye lid swelling and diplopia. Often, the possibility of an inflammatory process is raised. An excision biopsy often confirms the seemingly improbable diagnosis of an orbital metastasis. Orbital metastases remain an unfavorable prognostic factor, but the diagnosis and treatment are still important in order to prevent loss of vision and improve the patient's quality of life. As previous studies have shown, the overall survival rate is still as limited as nearly half a century ago. Ocular metastases have become less rare since the systemic treatment with chemotherapy prolongs survival in patients with cancer.

Breast cancer is primary cancer most frequently found in the case of orbital metastases (29-51\%) [9]. Lobular breast cancer represents the cancer subtype with the highest prevalence among orbital metastases. The high frequency of ILC in orbital metastases illustrates the special metastatic behavior of this tumor entity and may have implications for the understanding of the organotropism of metastatic lobular breast cancer [10].

We report the case of a patient who was discovered with orbital metastasis (OM) from an unknown breast cancer. This is a rare case of an orbital metastatic carcinoma preceding the diagnosis of a nonpalpable primary breast carcinoma. 
The importance of emergency treatment for rapidly progressing lesions is stressed as well as the need for detailed treatment planning to prevent possible damage to sensitive normal structures.

\section{Case Report}

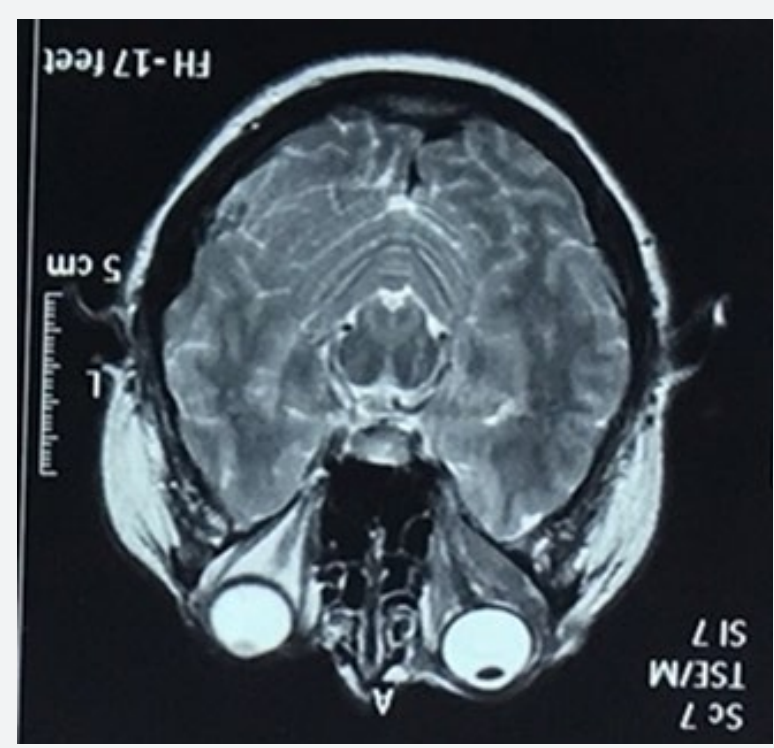

Figure 1: Pre-operative MRI of the orbit.

The initial pre-operative orbit MRI scans shows abnormal hypointense thickening of the episcleral tissues and extraocular muscles. The orbital fat also shows diffuse abnormal T2 hypointensity.

A 61-year-old woman presented with a history of gradually worsening blurred vision, periorbital swelling, pain and proptosis in the right eye. Magnetic resonance imaging (MRI) of the brain and orbits showed a diffuse abnormal enhancement involving the right orbit (Figure 1). The differential diagnosis included severe inflammatory response, pseudotumor, lymphoma, primary or metastatic neoplasia. The patient was referred to Division of Hematology to confirm a suspicion on lymphoma. The biopsy was submitted to the Ophthalmology Division where the possibility of lymphoma was excluded. Histologically, the biopsy demonstrated metastatic carcinoma of possible primary breast cancer estrogen receptor (ER) positive, progesterone receptor (PR) positive. The diagnosis of carcinoma was made; however, the site of origin of carcinoma was not yet determined. Chest and abdominal CT scan and mammography were negative. Since lymphoproliferative lesions are the most common primary orbital tumor in older adults ( $\geq 60$ years of age), representing a spectrum of disorders and radiologic examinations do not allow reliable differentiation between benign and malignant lymphoproliferative disorders, hematologists indicated right enucleation. The surgical procedure was eventually carried out. The pathology report of the enucleated eyeball identified a microscopic focus of accumulated carcinoma cells (small cell carcinoma) in the sclera. While, the pathology report of the biopsed orbital masses demonstrated hypercellular population of small cells that interdigitate between collagen bundles in a single line, so-called "Indian file". On immunohistochemical stains, the tumor cells from the orbital mass tissue were positive for ER, S100, CKW, CK AE1/AE3 and Lyzozim. These cells were negative for Plasma cell, Vimentin, TTF, PR, Melanoma, Melan A, LCA, SYN, Chromogranin, E-cadherin, CK 20, RCC, NSE and Actin. The findings were suggestive of metastatic lobular breast cancer. Post-operative MRI of the orbits demonstrated an expansile extraconal-intraconal mass that involved retrobulbar fat, eyelid, extraocular muscles, right nasolacrimal duct and right optic nerve (Figure 2). Postoperative chest CT scan revealed inhomogeneous loose tissue in left armpit. Cytological FNAB (fine needle aspiration biopsy) finding was negative for atypical cells. Some forms of invasive lobular carcinoma (ILC) do not present with a palpable mass as ILC is less likely to be associated with a fibrous tissue reaction. No further diagnostic studies were taken to rule out primary breast cancer (MRI, digital tomosynthesis) or primary lung cancer (bronchoscopy).

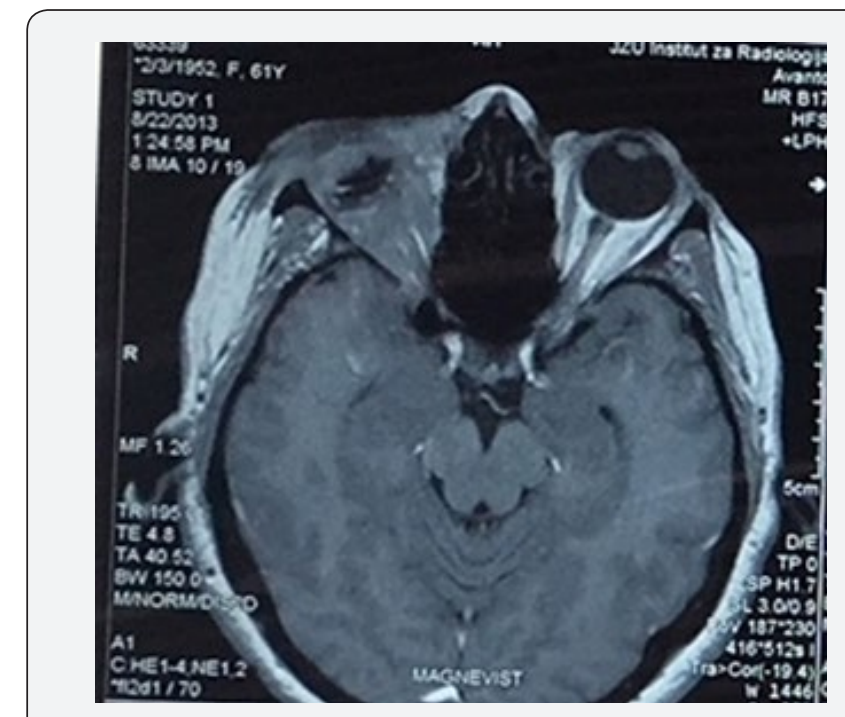

Figure 2: Post-operative MRI of the orbit.

Post-enucleation axial T1-weighted MR image demonstrates abnormal enhancement of the retrobulbar fat and extraocular muscles.

We believe that variability in the diagnostic report provided by pathologist was probably due to the common morphological features of the lobular carcinoma and small cell carcinoma. Both tumor cell lines are small, morphologically. Tumor cell of lobular carcinoma tends to have a loss of expression of E-cadherin. Small cell cancer tumors usually express chromogranin A and synaptophysin in $>50 \%$ of the cell population. Small cell breast carcinoma is a very rare (the literature describes $<40$ cases), yet highly aggressive variant of a neuroendocrine carcinoma. The first challenge in the proper diagnosis of small cell carcinoma of the breast is to determine whether it is breast primary, or whether it is in fact a 'secondary' site metastized from another cancer elsewhere in the body, (and the lung would be the first place to look). It is impossible to distinguish metastatic and primary small 
cell breast carcinomas on the basis of histological evaluation. Treatment by paclitaxel, cisplatin and etoposide have shown to be highly effective against the spread and recurrence of small cell breast carcinoma. Finally, based on the pathologist's examination and diagnostic radiology exams, first-line systemic chemotherapy of metastatic extrapulmonary small cell carcinoma was initiated using Cisplatin 75mg/m2 and Paclitaxel 175mg/m2 (x 6 cycles) and sequential external beam radiotherapy, particularly 3D conformal radiotherapy to the orbit to a cumulative dose of 3000cGy.

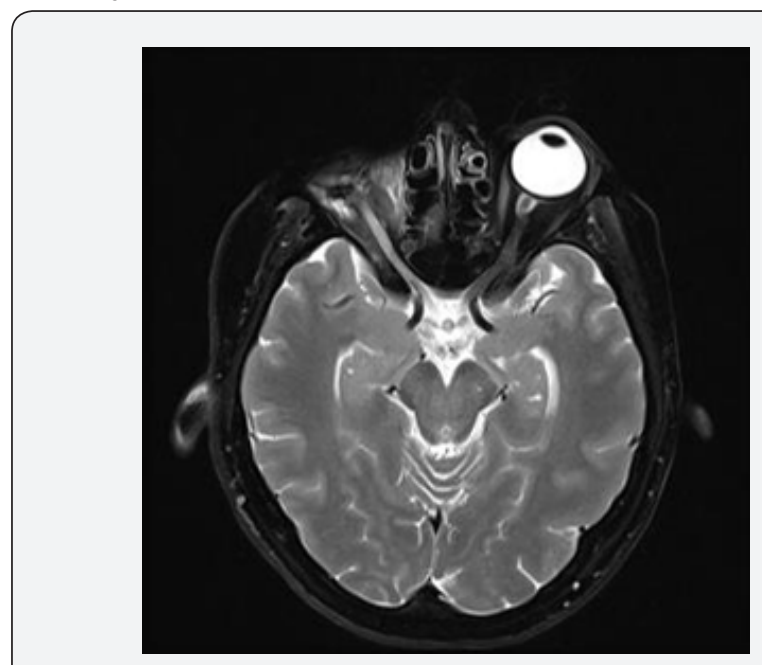

Figure 3: Post-radiation MRI of the orbit.

Axial T2- weighted MR image shows almost complete resolution of the mass on the right.

One year after the initial diagnose she found a lump in the left breast and in the left underarm area. Based on the mammogram report and FNA cytology, she underwent through left modified radical mastectomy (Histologic type: ILC, pTNM pT2 size of carcinoma 3.5cm pN3 19/19 extranodal extension G3 L1 Stage IIIC, hormone receptor status ER 3+ 95\%, PR - 5\% Her2/neu 2+, SISH negative, Ki 67 1+ 15\%, p53 - 5\%) and was started on hormone therapy with aromatase inhibitor. The post-radiation MRI of the orbits demonstrated almost complete radiologic response (Figure 3). On a follow-up visit 36 months after her breast surgery, she was disease free. Three months later, computed tomography of the abdomen revealed features of both small and large bowel obstruction with moderate ascites, left hydronephrosis with ureteral obstruction and gastric wall thickening. Arrangements for palliation were made. A ileostomy was performed. She passed away 4 years after her initial orbital manifestation.

\section{Discussion}

Orbital metastasis is relatively uncommon. In general patients with metastatic disease do not develop orbital metastases, being identified clinically in less than $1 \%$ of cases. Orbital symptoms occur well before local symptoms from the primary tumour manifest, and as such are the first indication of advanced malignancy $[11,12]$. In contrast to IDC metastasis to the CNS, which tends to form metastatic masses in the brain parenchyma, ILC that metastasizes to the CNS has a strikingly high propensity to spread diffusely along the leptomeninges. Diffuse infiltrative enhancing soft tissue replacing the postseptal fat or connective tissue often accompanies the abnormally enhancing extraocular muscles. This diffuse infiltrative expansile process often causes proptosis. The Tenon capsule, sclera, and eyelid soft tissue may also be involved [13-16].

Common tumours that have a predilection to metastasise to the orbit include breast cancer, bronchogenic cancer, prostate cancer, gastrointestinal adenocarcinoma, thyroid carcinoma, renal cell carcinoma, neuroblastoma, Ewing sarcoma and Wilms tumour [9$12,17,18]$. Of all metastatic tumors to the orbit, breast carcinoma is considered to be the most prevalent primary tumor, accounting for $29 \%$ to $70 \%$ of all metastases [17]. It is possible that the actual frequency of breast cancer orbital metastases is much higher than that indicated in the published series. Metastatic involvement of ocular structures in breast cancer seems to be a rare clinical entity; nevertheless, autopsy histopathological inquiries propose that $10-37 \%$ of patients with breast cancer have detectable ocular or orbital metastasis [19]. Still many breast cancer orbital metastases remain subclinical and are never diagnosed [20].

Infiltrating lobular breast cancer is a special breast cancer subtype. It accounts for $10-15 \%$ of all mammary carcinomas and for $\sim 1 \%$ of all malignancies [21]. ILCs display a distinct histomorphology and are almost always estrogen receptor (ER) positive [22]. The absence of the PR in luminal subtypes of breast cancer has a higher risk of relapse and lower disease-free survival $[23,24]$. This is in part due to an increase in hormone resistance among PR negative breast cancers [25]. PR negative lobular carcinoma confers a particularly poor response to hormonal therapy, and thus, a worse prognosis. ILC growth is strongly dependent on estrogenic stimulation [26]. The list of anatomical sites associated with ILC metastasis (ovaries, abdominal cavity, skin, bone) reads like a catalog of tissue compartments with a favorable steroid hormone supply. Estrogen concentrations are up to 1000 -fold higher in ovarian tissue and peritoneal cavity fluid as compared with the body circulation [27]. Moreover, estrogens are produced by mesenchymal cells of the dermis, adipose tissue and bone [28]. Accordingly, ILCs seem to metastasize to sites of estrogen production. The most convincing case supporting this notion has been documented by Arnould et al. [29] they have reported an ILC metastasis within an estrogen-producing granulosa cell tumor of the ovary, which had developed under tamoxifen therapy [29]. There is indirect evidence that the orbital fat pad produces steroid hormones to regulate tear film composition [30,31]. Alternatively, orbital metastasis from ILBC may simply extend to the orbit from nearby bone metastases or from occult meningeosis carcinomatosa. The mean interval from diagnosis of primary breast carcinoma to detection of orbital metastasis ranges from 4.5 to 6.5 years [32]. After the diagnosis, the prognosis is poor: in fact, the median survival is 31 months, the median is 19 months with a range of one to 116 months [33]. Ocular metastasis can represent the initial manifestation of breast carcinoma, with up to $26 \%$ of orbital breast metastases 


\section{Juniper Online Journal of Case Studies}

presenting before the discovery of the primary tumor [34]. The diagnosis of ocular metastases is based primarily on clinical findings supplemented by imaging studies (CT, MRI).

Orbital metastases originating from breast carcinoma predicts widespread metastatic disease in other organs. Systemic treatment of metastatic breast cancer may include some form of hormone therapy and/or chemotherapy depending on the overall disease burden [35]. Enucleation offers no advantage concerning disease progression or survival [32]. Orbital surgery is primarily used for diagnostic rather than therapeutic purposes given that the disease is usually widespread at time of diagnosis and is not curative. In selected cases, tumor resection, even if incomplete, may be appropriate to improve symptoms of pain, diplopia, and proptosis. Treatment is primarily with radiotherapy, typically with a total dose of 20 - 40Gy [11]. It is usually administered to control tumor growth, preserve visual function in the short term, decrease proptosis and exposure keratopathy, or to improve patient comfort [32].

\section{Conclusion}

In summary, although rare, orbital metastasis can be the initial and sole presenting feature of breast cancer. It can occur in the presence of normal breast examination or negative mammogram. It can be misdiagnosed as orbital pseudotumour. Patients with ocular symptoms such as ptosis, proptosis, diplopia, pain, exophthalmus, biopsy and histopathological examination of the orbital lesion should be considered, particularly in elderly. This case demonstrates the importance of the possibility of dual pathology, which may lead to diagnostic confusion. The pattern of ILC metastasis emphasizes its presentation at unusual sites such as the leptomeninges and orbit. This knowledge may aid in accurate imaging interpretation and treatment planning in patients with metastatic ILC of the breast.

\section{Declaration of Authorship}

EL and SS drafted the manuscript and performed data collection and interpretation. EL and ZA conceived the study and made critical revision of the manuscript. VK, VK and MI made critical revision of the manuscript. All authors of this paper have read and approved the final version submitted.

\section{Competing Interests}

All authors have completed the Unified Competing Interest form at www.icmje.org/coi_disclosure.pdf (available on request from the corresponding author) and declare: no support from any organization for the submitted work; no financial relationships with any organizations that might have an interest in the submitted work in the previous 3 years; no other relationships or activities that could appear to have influenced the submitted work.

\section{References}

1. Martinez V, Azzopardi JG (1979) Invasive lobular carcinoma of the breast: incidence and variants. Histopathology 3(6): 467-488.
2. Borst MJ, Ingold JA (1993) Metastatic patterns of invasive lobular versus invasive ductal carcinoma of the breast. Surgery 114(4): 637-641.

3. Harris M, Howell A, Chrissohou M, Swindell RI, Hudson M, (1984) A comparison of the metastatic pattern of infiltrating lobular carcinoma and infiltrating duct carcinoma of the breast. Br J Cancer 50(1): 23-30.

4. Ferlicot S, Vincent-salomon A, Médioni J, Genin P, Rosty C, et al. (2004) Wide metastatic spreading in infiltrating lobular carcinoma of the breast. Eur J Cancer 40(3): 336-341.

5. Lamovec J, Bracko M (1991) Metastatic pattern of infiltrating lobular carcinoma of the breast: an autopsy study. J Surg Oncol 48(1): 28-33.

6. Winston CB, Hadar O, Teitcher JB, Caravelli JF, Sklarin NT, et al. (2000) Metastatic lobular carcinoma of the breast: patterns of spread in the chest, abdomen, and pelvis on CT. AJR Am J Roentgenol 175(3): 795800 .

7. Arpino G, Bardou VJ, Clark GM, Elledge RM (2004) Infiltrating lobular carcinoma of the breast: tumor characteristics and clinical outcome. Breast Cancer Res 6(3): R149-R156.

8. Berg JW, Hutter RV (1995) Breast cancer. Cancer 75(1 Suppl): 257-269.

9. Cornelis F, Mejdoubi M, Dousset V (2007) Bilateral orbital extension of breast cancer metastasis. J Radiol 88(5 Pt 1): 684-686.

10. Raap M, Antonopoulos W, Dämmrich M, Christgen H, Steinmann D, et al. (2015) High frequency of lobular breast cancer in distant metastases to the orbit. Cancer Med 4(1): 104-111.

11. Char DH, Miller T, Kroll S (1997) Orbital metastases: diagnosis and course. Br J Ophthalmol 81(5): 386-390.

12. Green S, Som PM, Lavagnini PG (1995) Bilateral orbital metastases from prostate carcinoma: case presentation and CT findings. AJNR Am J Neuroradiol 16(2): 417-419.

13. Toller KK, Gigantelli JW, Spalding MJ (1998) Bilateral orbital metastases from breast carcinoma. A case of false pseudotumor. Ophthalmology 105(10): 1897-1901.

14. Gupta S, Bhatt VR, Varma S (2011) Unilateral orbital pain and eyelid swelling in a 46-year-old woman: orbital metastasis of occult invasive lobular carcinoma of breast masquerading orbital pseudotumour. BMJ Case Rep 2011.

15. Tomizawa Y, Ocque R, Ohori NP (2012) Orbital metastasis as the initial presentation of invasive lobular carcinoma of breast. Intern Med 51(12): 1635-1638.

16. Zhang GJ, Adachi I, Yin DF, Narabayashi M, Tokue Y, et al. (1995) Eyelid metastasis from breast cancer showing marked response to chemotherapy. Jpn J Clin Oncol 25(1): 10-15.

17. Shields JA, Shields CL, Scartozzi R (2004) Survey of 1264 patients with orbital tumors and simulating lesions: The 2002 Montgomery Lecture, part 1. Ophthalmology 111(5): 997-1008.

18. Holland D, Maune S, Kovács G, Behrendt S (2003) Metastatic tumors of the orbit: a retrospective study. Orbit 22(1): 15-24.

19. Merrill CF, Kaufman DI, Dimitrov NV (1991) Breast cancer metastatic to the eye is a common entity. Cancer 68(3): 623-627.

20. Dieing A, Schulz CO, Schmid P, Roever AC, Lehenbauer-Dehm S, et al. (2004) Orbital metastases in breast cancer: report of two cases and review of the literature. J Cancer Res Clin Oncol 130(12): 745-748.

21. Weigelt B, Reis-Filho JS (2009) Histological and molecular types of breast cancer: is there a unifying taxonomy? Nat Rev Clin Oncol 6(12): 718-730.

22. Walker RA, Hanby A, Pinder SE, Thomas J, Ellis IO (2012) Current issues in diagnostic breast pathology. J Clin Pathol 65(9): 771-785. 
23. Cancello G, Maisonneuve P, Rotmensz N, Viale G, Mastropasqua MG, et al. (2013) Progesterone receptor loss identifies Luminal B breast cancer subgroups at higher risk of relapse. Ann Oncol 24(3): 661-668.

24. Feeley LP, Mulligan AM, Pinnaduwage D, Bull SB, Andrulis IL (2014) Distinguishing luminal breast cancer subtypes by Ki67, progesterone receptor or TP53 status provides prognostic information. Mod Pathol 27(4): 554-561.

25. Linke SP, Bremer TM, Herold CD, Sauter G, Diamond C (2006) A multimarker model to predict outcome in tamoxifen-treated breast cancer patients. Clin Cancer Res 12(4): 1175-1183.

26. Rakha EA, El-sayed ME, Powe DG, Green AR, Habashy H, et al. (2008) Invasive lobular carcinoma of the breast: response to hormonal therapy and outcomes. Eur J Cancer 44(1): 73-83.

27. Koninckx PR, Kennedy SH, Barlow DH (1998) Endometriotic disease: the role of peritoneal fluid. Hum Reprod Update 4(5): 741-751.

28. Simpson E, Rubin G, Clyne C, Robertson K, O’Donnell L, et al. (1999) Local estrogen biosynthesis in males and females. Endocr Relat Cancer 6(2): 131-137.

29. Arnould L, Franco N, Soubeyrand MS, Mege F, Belichard C, et al. (2002) Breast carcinoma metastasis within granulosa cell tumor of the ovary: morphologic, immunohistologic, and molecular analyses of the two different tumor cell populations. Hum Pathol 33(4): 445-448.
30. Spelsberg H, Klueppel M, Reinhard T, Glaeser M, Niederacher D, et al. (2004) Detection of oestrogen receptors (ER) alpha and beta in conjunctiva, lacrimal gland, and tarsal plates. Eye (Lond) 18(7): 729-733.

31. Turaka K, Nottage JM, Hammersmith KM, Nagra PK, Rapuano CJ (2013) Dry eye syndrome in aromatase inhibitor users. Clin Experiment Ophthalmol 41(3): 239-243.

32. Ahmad SM, Esmaeli B (2007) Metastatic tumors of the orbit and ocular adnexa. Curr Opin Ophthalmol 18(5): 405-413.

33. Garrity JA, Henderson JW, Cameron JD. Henderson's Orbital Tumors. 4th ed. New York, NY: Raven Press; 2007. Metastatic carcinomas; pp. 313-326

34. Goldberg RA, Rootman J, Cline RA (1990) Tumors metastatic to the orbit: a changing picture. Surv Ophthalmol 35(1): 1-24.

35. Wickremasinghe S, Dansingani KK, Tranos P, Liyanage S, Jones A, et al. (2007) Ocular presentations of breast cancer. Acta Ophthalmol Scand 85(2): 133-142.

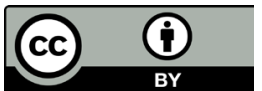

This work is licensed under Creative Commons Attribution 4.0 License DOI: 10.19080/JOJCS.2019.10.555797 